\title{
Regulatory Mechanism of MicroRNA-145 in the Pathogenesis of Acute Aortic Dissection
}

\author{
Tianbo Li, Chencheng Liu, Lingchao Liu, Han Xia, Yingbin Xiao, Xuefeng Wang, and Yong Wang \\ Department of Cardiovascular Surgery, the Second Affiliated Hospital (Xinqiao Hospital) of Chinese People's Liberation Army Medical University, \\ Chongqing, China.
}

Purpose: Previous studies have confirmed that microRNAs play important roles in the pathogenesis of acute aortic dissection (AAD). Here, we aimed to explore the role of miR-145 and its regulatory mechanism in the pathogenesis of AAD.

Materials and Methods: AAD tissue samples were harvested from patients with aortic dissection and normal donors. Rat aortic vascular smooth muscle cells (VSMCs) were transfected with miR-145 mimic/inhibitor or negative control mimic/inhibitor. Gene and protein expression was measured in human aortic dissection tissue specimens and VSMCs by qRT-PCR and Western blot. Luciferase reporter assay was applied to verify whether connective tissue growth factor (CTGF) was a direct target of miR-145 in VSMCs. Methyl thiazolyl tetrazolium assay was used to detect VSMC viability.

Results: miR-145 expression was downregulated in aortic dissection tissues and was associated with the survival of patients with AAD. Overexpression of miR-145 promoted VSMC proliferation and inhibited cell apoptosis. Moreover, CTGF, which was increased in aortic dissection tissues, was decreased by miR-145 mimic and increased by miR-145 inhibitor. Furthermore, CTGF was confirmed as a target of miR-145 and could reverse the promotion effect of miR-145 on the progression of AAD.

Conclusion: miR-145 suppressed the progression of AAD by targeting CTGF, suggesting that a miR-145/CTGF axis may provide a potential therapeutic target for AAD.

Key Words: miR-145, acute aortic dissection, progression, CTGF

\section{INTRODUCTION}

Aortic dissection occurs when a rupture in the intima of the aorta enables blood to damage the tunica media, leaving the vascular layer vulnerable to separation and degeneration, and is one of the most dangerous vascular diseases. ${ }^{1,2}$ Despite tremendous progress in treatment, aortic dissection remains a clinical medical emergency that requires rapid intervention to avoid death.

\footnotetext{
Received: October 15, 2018 Revised: February 16, 2019

Accepted: February 18, 2019

Corresponding author: Yong Wang, MD, Department of Cardiovascular Surgery, the Second Affiliated Hospital (Xinqiao Hospital) of Chinese People's Liberation Army Medical University, NO. 183, Xinqiao Zhengjie, Shapingba district, Chongqing 400037, China.

Tel: 86-23-68774107, Fax: 86-23-68774107, E-mail: wangrong1111@126.com

-The authors have no potential conflicts of interest to disclose.

(C) Copyright: Yonsei University College of Medicine 2019

This is an Open Access article distributed under the terms of the Creative Commons Attribution Non-Commercial License (https://creativecommons.org/licenses/ by-nc/4.0) which permits unrestricted non-commercial use, distribution, and reproduction in any medium, provided the original work is properly cited.
}

MicroRNAs (miRNAs), non-coding RNAs of 21 to 25 nucleotides in length, are reported to regulate gene expression posttranscriptionally via degrading mRNA or repressing translation. ${ }^{3}$ miRNAs have been reported to play important roles in regulating the development of many diseases, such as cell proliferation, differentiation, migration, and apoptosis. ${ }^{4}$ Recently, mounting evidence has demonstrated that miRNAs play important roles not only in tumor progression, but also in cardiovascular diseases. ${ }^{5,6}$ For instance, miR-21 was shown to attenuate diabetic cardiomyopathy via targeting gelsolin. ${ }^{7}$ miR-33 was found to be up-regulated in atherosclerosis and involved in its progress, representing a novel target for atherosclerosis treatment. ${ }^{8}$ Furthermore, Yang, et al. ${ }^{9}$ showed that decreasing miR-327 expression exerts a cardio-protective effect against myocardial ischemia/reperfusion injury. In aortic dissection, miR-17 was reported to be expressed at lower levels in thoracic aortic dissection and to be associated with up-regulated RUNX1. ${ }^{10}$ Huang and his colleagues ${ }^{11}$ showed that knockdown of miR-21 exacerbates thoracic aortic aneurysm and dissection via TGF- $\beta$ /SMAD3 signaling pathway. Dong, et al. ${ }^{12}$ re- 
ported that miR-15a and miR-23a were highly expressed in acute aortic dissection (AAD) and that they have good clinical value for the diagnosis of AAD. Also, miR-4787 and miR-4306 were described as potential biomarkers for the diagnosis of $\mathrm{AAD}$ and as being involved in its pathogenesis. ${ }^{13}$ Moreover, $\mathrm{Li}$, et al. ${ }^{14}$ reported that miR-145 expression was decreased in AAD. However, its role and regulatory mechanism in the pathogenesis of $\mathrm{AAD}$ remains unclear.

Experiments conducted by many researchers have shown that connective tissue growth factor (CTGF), a matricellular protein belonging to the transforming growth factor beta super family, is induced in the heart following cardiac injury ${ }^{15}$ CTGF is expressed lowly in the rat aorta and is involved in rescuing aorta remodeling in rat models of aortic dissection. ${ }^{16}$ Frangogiannis ${ }^{17}$ showed that CTGF was involved in the proliferation and apoptosis of vascular smooth muscle cells (VSMCs). However, whether CTGF participates in the pathogenesis of AAD regulated by miR-145 has not been reported until now.

Our study was conducted to investigate miR-145 and CTGF expression in patients with $\mathrm{AAD}$ and to explore the role and mechanisms of miR-145 in regulating the development of AAD in VSMCs. We found that miR-145 is downregulated in $\mathrm{AAD}$ and that overexpression of miR-145 promotes the progression of AAD by targeting CTGF.

\section{MATERIALS AND METHODS}

\section{Clinical samples and ethics statement}

Sixty samples of the ascending aorta in patients with AAD (28 type) and aortic valve disease (32 aortic valve replacement) were obtained during surgery. Aortic samples from donors for heart transplants were used as normal controls. AAD was classified according to the Stanford classification. None of the included patients underwent preoperative therapy prior to enrollment. Exclusion criteria were Marfan syndrome, Ehlers-Danlos syndrome, family-type thoracic and abdominal aortic dissection, and aortic-related lesions, such as aortitis. Table 1 details the clinical characteristics of the study population. Follow-up evaluations were conducted for all included patients with $\mathrm{AAD}$, and the follow-up time was determined from the surgery date to 10 years. The number of patients at risk was 28 . The medium expression of miR-145 was the cutoff between higher and lower miR-145 groups. This study was approved by the Ethics Review Committee of the Second Affiliated Hospital (Xinqiao Hospital) of Chinese People's Liberation Army Medical University (IBR No. XQYY-LUNLI-FJ001.02) and conducted in accordance with its instructions. Written informed consent was provided by all patients. Tissue samples were kept in liquid nitrogen and stored at $-80^{\circ} \mathrm{C}$ for qRT-PCR and Western blot analysis.

\section{Cell culture and cell transfection}

We purchased rat aortic VSMC line from the cell bank of the
Chinese Academy of Sciences (Beijing, China). The cells were cultured in DMEM Medium (Thermo Fisher, Waltham, MA, USA) with $10 \%$ fetal bovine serum at $37^{\circ} \mathrm{C}$ in a humidified $5 \%$ $\mathrm{CO}_{2}$ incubator. The cultured VSMCs were transfected with miR-145 mimic or inhibitor to overexpress or silence miR-145 following the manufacturer's instruction. The control VSMCs were transfected with negative control mimic or inhibitor. After infection for $48 \mathrm{hr}$, VSMCs were harvested for analysis of miR-145 expression and CTGF levels.

\section{RNA isolation and qRT-PCR}

TRIzol reagent (Invitrogen, Carlsbad, CA, USA) was used to extract total RNA from VSMCs and aortic tissues according to the manufacturer's instructions. First-strand complementary DNA was generated using the Reverse-Transcription System Kit (Invitrogen). qRT-PCR was performed following a standard protocol using the ABI Prism 7500 system (Thermo Fisher). U6 and GAPDH served as an internal control. The $2^{-\Delta \Delta C t}$ method was used to determine expression changes represented as a relative fold to the control.

\section{Immunoblotting assay}

After treatment, cell lysates were subjected to $10 \%$ SDS-PAGE electrophoresis and transferred to polyvinylidene fluoride membranes, as described previously. After blocking with 5$10 \%$ skim milk in PBS for $2 \mathrm{hr}$ at room temperature, the primary antibodies (Caspase-8, CTGF and GAPDH) were added to incubate at $4^{\circ} \mathrm{C}$ overnight, respectively. Then, horseradish peroxidase conjugated secondary antibodies were added to incubate for $2 \mathrm{hr}$ at room temperature. Finally, the enhanced chemiluminescence kit (ECL, Millipore, BB-3501, Ameshame, UK) was used to detect the signals. Equal loading of protein in each lane was confirmed by probing with GAPDH antibody.

\section{Methyl thiazolyl tetrazolium assay}

Methyl thiazolyl tetrazolium (MTT) was used to detect cell viability. $5 \times 10^{3}$ VSMCs with different transfection were added into 96 -well plates, respectively, and cultured for $48 \mathrm{hr}$ at $37^{\circ} \mathrm{C}$ with $5 \% \mathrm{CO}_{2}$. Then MTT medium $(20 \mu \mathrm{L})$ was added to each well. After incubation at $37^{\circ} \mathrm{C}$ for $4 \mathrm{hr}$, the MTT medium was pipetted out, and $100 \mu \mathrm{L}$ of DMSO was added for an additional $10 \mathrm{~min}$. Finally, the plates were read at a wavelength of $490 \mathrm{~nm}$ at $12,24,36$, and $48 \mathrm{hr}$ to measure the absorbance of each well.

\section{Dual luciferase reporter assay}

The recombinant pMIR-reportor luciferase vector was applied for luciferase assays. miR-145 mimic and pMIR-reportor luciferase vector containing 3'-UTR of wild-type (WT) or mutanttype (MUT) CTGF were co-transfected into the rat VSMCs following the Lipofectamine 2000 (Thermo Fisher) instructions. The Dual Luciferase Reporter Assay System (Promega, Madison, WI, USA) was then used to measure the luciferase activity. 


\section{Statistical analysis}

Experimental values are expressed as the mean \pm SD of at least three independent experiments. When a significant difference was detected, the Mann-Whitney U test or Tukey's post hoc test after one-way ANOVA was applied. SPSS v.19.0 (IBM Corp., Armonk, NY, USA) and GraphPad Prism 5.02 (GraphPad Software Inc., La Jolla, CA, USA) were used to perform statistical analyses and complete graph presentations. The logrank test and Kaplan-Meier analysis were applied to calculate the relevance between miR-145 expression and the postoperative survival time of patients with AAD. $p<0.05$ was considered to indicate a significant difference.

\section{RESULTS}

\section{Clinical characteristics of patients}

We enrolled 60 patients with AAD and 60 controls in this study. The clinical characteristics of the patients are listed in Table 1.

Table 1. Clinical Characteristics of the $A A D$ and Control Groups

\begin{tabular}{lcc}
\hline Characteristics & AAD (n=60) & Control ( $\mathbf{n = 6 0})$ \\
\hline Age (yr) & $51.93 \pm 8.87$ & $52.21 \pm 8.21$ \\
Male & $49(81.6)$ & $47(78.3)$ \\
Smoker & $24(40)$ & $8(13.3)$ \\
Alcoholic & $20(33.3)$ & $4(6.7)$ \\
Hypertension & $45(75)^{*}$ & 0 \\
\hline Atherosclerosis & $25(41.7)$ & $19(31.7)$ \\
Aortic & $3(5)$ & 0 \\
Cocaine abuse & 0 & 0 \\
Diabetes & $1(1.7)$ & $1(1.7)$ \\
Bicuspid aortic & 0 & 0 \\
\hline
\end{tabular}

$A A D$, acute aortic dissection.

Data are presented as a mean \pm SD or $n(\%)$.

${ }^{*} p<0.05$.
There were no statistically significant differences in clinical characteristics between the $\mathrm{AAD}$ and control groups, except for hypertension. Most AAD patients (75\%) had concomitant hypertension, while normal patients had no hypertension.

\section{miR-145 is downregulated in AAD specimens}

To investigate the role of miR-145 in the pathogenesis of AAD, we first detected the gene expression of miR-145 in AAD specimens. As shown in Fig. 1A, miR-145 expression was lower in aortic dissection specimens than in normal aorta specimens. Supplementary Table 1 (only online) showed the raw data of the distribution of miR-145 in patients. Moreover, we explored the relationship between miR-145 and the survival time of patients. The results showed that the higher the expression of miR-145, the higher the survival rate of aortic dissection patients (Fig. 1B).

\section{miR-145 inhibits the progression of AAD}

Secondly, miR-145 mimic or inhibitor was utilized to elicit overexpression or silencing of miR-145 expression in VSMCs. As we expected, miR-145 expression was significantly increased in VSMCs transfected with miR-145 mimic, but decreased in those transfected with miR-145 inhibitor, compared with the negative control (Fig. 2A). MTT results showed that over-expression of miR-145 facilitated cell viability, while silencing miR-145 inhibited cell viability (Fig. 2B). Moreover, Western blot and qRT-PCR results showed that miR-145 mimic inhibited the expression of Caspase-8, whereas miR-145 inhibitor enhanced its expression (Fig. 2C and D). All the results showed that miR-145 promotes VSMC proliferation and inhibits cell apoptosis, suggesting an inhibitory effect on the progression of AAD.

\section{CTGF is a target of miR-145 in AAD}

CTGF was predicted as a potential target of miR-145 by Tar-

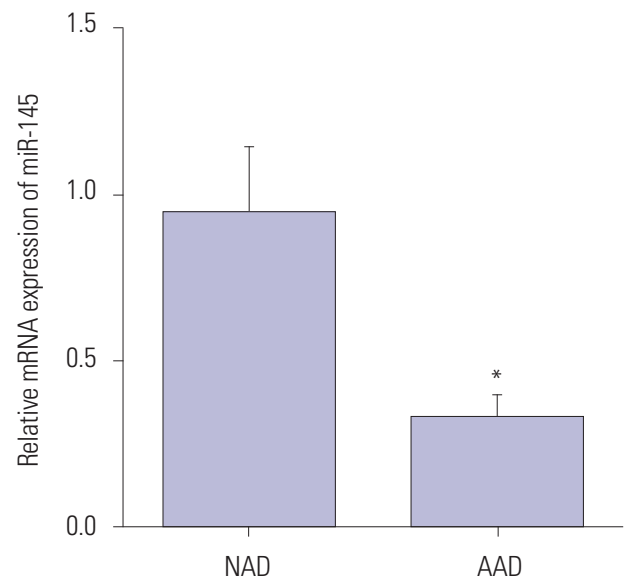

A

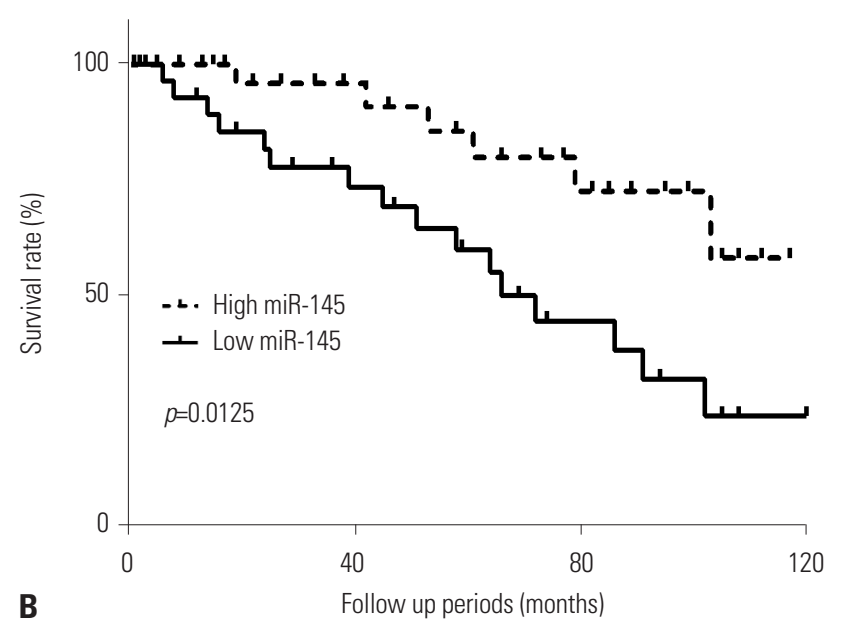

Fig. 1. miR-145 was decreased in human AAD specimens. (A) qRT-PCR results revealed decreased expression of miR-145 in $A A D$ specimens, compared with NAD specimens $(n=60)$. (B) Kaplan-Meier survival analysis showed a negative correlation of miR-145 with the survival time of aortic dissection patients $(\mathrm{n}=60)$. * $p<0.05$. AAD, acute aortic dissection; NAD, normal aortic dissection. 



Fig. 2. miR-145 mimic promotes VSMC proliferation and inhibits VSMC apoptosis. (A) miR-145 expression was increased in VSMCs transfected with miR145 mimic or decreased with miR-145 inhibitor. (B) VSMC viability increased after treating with miR-145 mimic or decreased after treating with miR-145 inhibitor. (C and D) Caspase-8 expression was inhibited by miR-145 mimic and promoted by miR-145 inhibitor in VSMCs according to Western blot and qRTPCR. ${ }^{*} p<0.05,{ }^{\dagger} p<0.01$. VSMC, vascular smooth muscle cell.

getScanHuman (www.targetscan.org/) (Fig. 3A). Luciferase reporter assay was carried out to further verify whether CTGF was the direct target of miR-145 in VSMCs, as Fig. 3B showed that CTGF 3'-UTR activity was decreased by miR-145 mimic in the wild group, while there was no significant change in the mutant group. Then, we measured CTGF expression by Western blot and qRT-PCR in AAD specimens. Results showed that CTGF was highly expressed in aortic dissection specimens in comparison with normal aorta (Fig. 3C and D). Furthermore, the relationship between CTGF and miR-145 measured by regression analysis was negative (Fig. 3E). Then, we detected CTGF expression by Western blot and qRT-PCR in VSMCs after transfection with miR-145 mimic or inhibitor. As we saw in Fig. 3F and G, CTGF expression was decreased by miR-145 mimic and increased by miR-145 inhibitor. All these data indicated that CTGF is a direct target of miR-145 in VSMCs.

\section{CTGF reverses miR-145 effects on the progression of AAD}

To assess the role of CTGF in AAD progression, we first silenced CTGF expression using CTGF siRNA. As we expected, CTGF expression was downregulated by CTGF siRNA (Fig. 4A). Then, we tested cell viability and cell apoptosis in VSMCs transfected with miR-145 inhibitor, CTGF siRNA, or both miR145 inhibitor and CTGF siRNA. As Fig. 4B showed, miR-145 inhibitor suppressed cell viability, while CTGF siRNA enhanced cell viability. Also, CTGF siRNA could reverse the inhibitory effect of miR-145 inhibitor on VSMC viability. Moreover, miR145 inhibitor enhanced Caspase-8 mRNA and protein expression, while CTGF siRNA inhibited its expression. Also, CTGF siRNA could reverse the promotion effect of miR-145 inhibitor on VSMC apoptosis (Fig. 4C and D). All these data showed that CTGF could reverse miR-145 effects on the progression of AAD.

\section{DISCUSSION}

The main conclusions of this study can be summarized as follows. miR-145 is downregulated in the aortic wall of patients with AAD and is associated with the survival of patients. However, CTGF is upregulated in aortic dissection specimens and appears to be a direct target of miR-145 in regulating the progression of aortic dissection in VSMCs.

As a post-transcriptional regulator of protein translation, the main role of miRNAs in aortic pathologies has been elucidated by several studies. In pathophysiological mechanisms, 


\section{CTGF 3'UTR-WT $\quad$ 5' ...CGAAUGACACUGUUCAGGAAUCG...3' hsa-miR-145-3p 3' '....UCUUGUCAUAAAGGUCCUUAGG...5'}

\section{A CTGF 3'UTR-MuT 5' ...CGAAUGACACUGUUCACCUAAGG...3'}
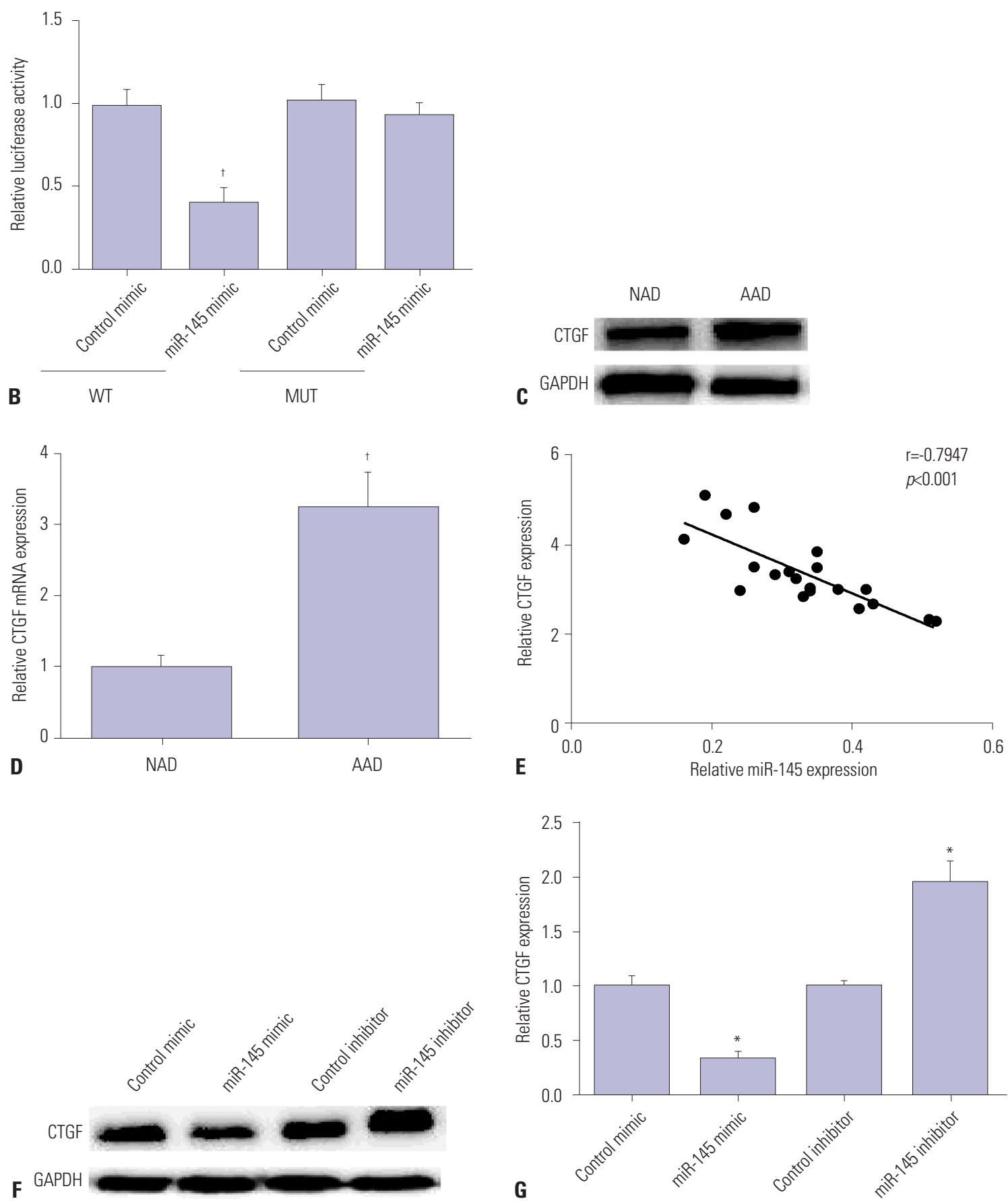

Fig. 3. CTGF is a target of miR-145 in VSMCs. (A) miR-145 was bound to the 3 'UTR of CTGF. (B) The luciferase activity of CTGF was decreased by miR-145 mimic in WT. (C) Western blot analysis showed higher expression of CTGF protein in AAD specimens than in NAD specimens. (D) qRT-PCR showed a higher expression of CTGF mRNA in AAD specimens than in NAD specimens. (E) Regression analysis showed a negative correlation of miR-145 with CTGF expression. (F) Western blot showed decreased or increased expression of CTGF protein in the miR-145 mimic or miR-145 inhibitor group, respectively. (G) qRT-PCR showed decreased or increased expression of CTGF gene in the miR-145 mimic or miR-145 inhibitor group, respectively. ${ }^{*} p<0.05,{ }^{\dagger} p<0.01$. CTGF, connective tissue growth factor; VSMC, vascular smooth muscle cell; $A A D$, acute aortic dissection; NAD, normal aortic dissection. 

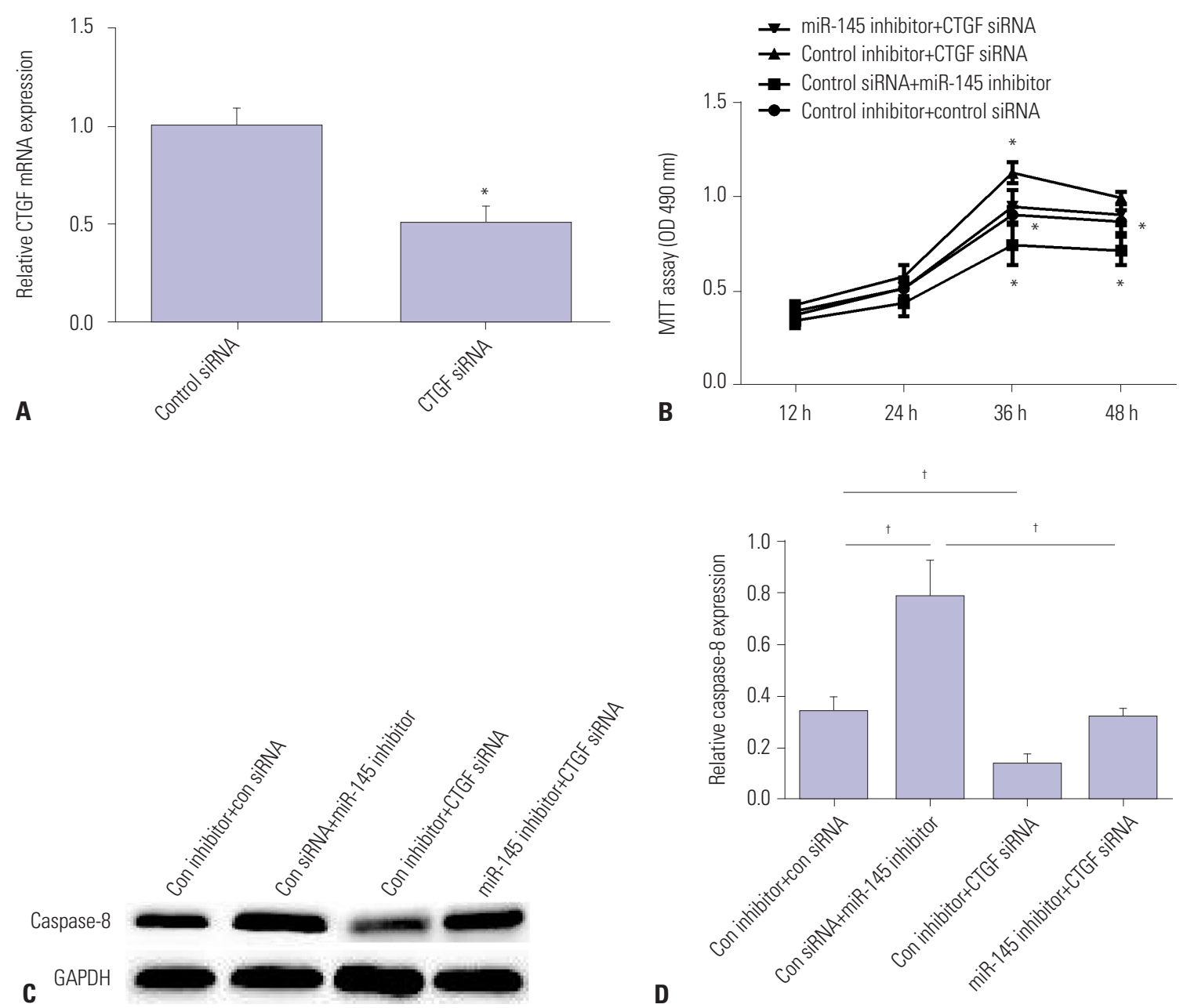

Fig. 4. CTGF attenuates miR-145 effects on VSMC proliferation and apoptosis. (A) qRT-PCR showed declined expression of CTGF in the CTGF siRNA group. (B) VSMC viability was detected by MTT for cells transfected with miR-145 inhibitor, CTGF siRNA, or both miR-145 inhibitor and CTGF siRNA. (C) Protein expression of Caspase- 8 was detected by Western blot after cells were transfected with miR-145 inhibitor, CTGF siRNA, or both miR-145 inhibitor and CTGF siRNA. (D) mRNA expression of Caspase-8 was detected by qRT-PCR after cells were transfected with miR-145 inhibitor, CTGF siRNA, or both miR-145 inhibitor and CTGF siRNA. ${ }^{*} p<0.05,{ }^{\dagger} p<0.01$. CTGF, connective tissue growth factor; VSMC, vascular smooth muscle cell.

miRNAs have become participants in cellular activity that leads to the formation of aneurysms, including inflammatory processes, degradation of extracellular matrix and loss of smooth muscle cells. ${ }^{18}$ For instance, downregulation of miR-29 was reported to attenuate aneurysm expansion, and miR-29 might represent a novel molecular target through which to maintain vascular wall structural integrity. ${ }^{19}$ miR-17 expression was increased in progressive aortic dilation by regulating TIMP- $1 .{ }^{20}$ Also, Elia, et al. ${ }^{21}$ showed that miR-143/145 expression was decreased in acute and chronic vascular stress and that it has a critical role during VSMC differentiation, strongly suggesting its involvement in the reversion of the VSMC differentiation phenotype that occurs during vascular disease. Moreover, miR-181b was found to be overexpressed in atherosclerosis and abdominal aortic aneurysms and to regulate their progression through regulating TIMP- 3 and Elastin. ${ }^{22}$

Aortic aneurysm and aortic dissection are two separate, but closely related entities. Compared with extensive studies on aortic aneurysms, studies on the role of miRNAs in AAD development are still limited. For example, miR-320 and miR-582 have been shown to serve as putative biomarkers for aortic dissection and to regulate VSMC apoptosis. ${ }^{23}$ Meanwhile, miR4787 and miR-4306 exhibit diagnostic value in patients with AAD ${ }^{13}$ In our study, we found that miR-145 was downregulated in aortic dissection specimens, which is consistent with research reported by Li, et al. ${ }^{14}$ We also noted that miR-145 enhanced VSMC proliferation and inhibited cell apoptosis. Our results provide evidence suggesting that overexpression of miR-145 suppresses the pathogenesis of aortic dissection.

In different tissues, several genes are thought to be the targets of miR-145. CTGF is also a predicted target of miR-145 according to the TargetScan bioinformatics-based database. In the present study, dual luciferase reporter assay confirmed that CTGF is a target of miR-145 in rat VSMCs and negatively regulates CTGF expression.

Recently, a large number of studies have found that CTGF is 
closely related to the occurrence and development of fibrosis in many tissues and organs, which is a complex pathophysiological process, including the blood vessels, skin, heart, lung, and liver. ${ }^{24-28}$ In our study, we discovered that CTGF is upregulated in aortic dissection specimens and is involved in the progression of aortic dissection. Zhou, et al. ${ }^{29}$ reported that CTGF served as a target of miR-503 in regulating cardiac fibrosis. Also, Wei, et al. ${ }^{30}$ showed that miR-26a targets CTGF to regulate cardiac fibrosis via NF- $\kappa \mathrm{B}$ signaling pathway. However, this is the first time that CTGF has been reported as the target of miR-145 in regulating the progression of AAD.

Taken together, our results indicate that overexpression of miR-145 suppresses the development of AAD possibly through inhibiting the expression of CTGF in the aortic wall. This study provides new insights into the molecular mechanisms of aortic dissection and may open a door for utilizing miRNAs in preventive strategies for major arterial dissection.

\section{ACKNOWLEDGEMENTS}

This study was supported by grants from the Project of Chongqing Science Committee (NO. csts2015shmszx120088).

\section{AUTHOR CONTRIBUTIONS}

Conceptualization: Yong Wang. Data curation: Tianbo Li. Formal analysis: Lingchao Liu. Funding acquisition: Yong Wang. Investigation: Han Xia. Methodology: Xuefeng Wang. Project administration: Tianbo Li. Resources: Yong Wang. Software: Yingbin Xiao. Supervision: Han Xia. Validation: Yingbin Xiao. Visualization: Xuefeng Wang. Writing_original draft: Tianbo Li. Writing_review \& editing: Chencheng Liu.

\section{ORCID iDs}

Tianbo Li Chencheng Liu Lingchao Liu Han Xia Yingbin Xiao Xuefeng Wang Yong Wang

\section{REFERENCES}

1. Rampoldi V, Trimarchi S, Eagle KA, Nienaber CA, Oh JK, Bossone E, et al. Simple risk models to predict surgical mortality in acute type A aortic dissection: the International Registry of Acute Aortic Dissection score. Ann Thorac Surg 2007;83:55-61.

2. Hagan PG, Nienaber CA, Isselbacher EM, Bruckman D, Karavite DJ, Russman PL, et al. The International Registry of Acute Aortic Dissection (IRAD): new insights into an old disease. JAMA 2000; 283:897-903.

3. Bartel DP. MicroRNAs: genomics, biogenesis, mechanism, and function. Cell 2004;116:281-97.

4. Sen CK, Ghatak S. miRNA control of tissue repair and regeneration. Am J Pathol 2015;185:2629-40.
5. Peng L, Chun-guang Q, Bei-fang L, Xue-zhi D, Zi-hao W, Yun-fu L, et al. Clinical impact of circulating miR-133, miR-1291 and miR-663b in plasma of patients with acute myocardial infarction. Diagn Pathol 2014;9:89.

6. Lee S, Choi E, Cha MJ, Hwang KC. Looking into a conceptual framework of ROS-miRNA-atrial fibrillation. Int J Mol Sci 2014;15: 21754-76.

7. Dai B, Li H, Fan J, Zhao Y, Yin Z, Nie X, et al. MiR-21 protected against diabetic cardiomyopathy induced diastolic dysfunction by targeting gelsolin. Cardiovasc Diabetol 2018;17:123.

8. Sun Y, Zhang D, Liu X, Li X, Liu F, Yu Y, et al. Endoplasmic reticulum stress affects lipid metabolism in atherosclerosis via CHOP activation and over-expression of miR-33. Cell Physiol Biochem 2018;48:1995-2010.

9. Yang Y, Yang J, Liu XW, Ding JW, Li S, Guo X, et al. Down-regulation of miR-327 alleviates ischemia/reperfusion-induced myocardial damage by targeting RP105. Cell Physiol Biochem 2018;49:1049-63.

10. Sun J, Chen G, Jing Y, He X, Dong J, Zheng J, et al. LncRNA expression profile of human thoracic aortic dissection by high-throughput sequencing. Cell Physiol Biochem 2018;46:1027-41.

11. Huang X, Yue Z, Wu J, Chen J, Wang S, Wu J, et al. MicroRNA-21 knockout exacerbates angiotensin II-induced thoracic aortic aneurysm and dissection in mice with abnormal transforming growth factor- $\beta$-SMAD3 signaling. Arterioscler Thromb Vasc Biol 2018;38: 1086-101.

12. Dong J, Bao J, Feng R, Zhao Z, Lu Q, Wang G, et al. Circulating microRNAs: a novel potential biomarker for diagnosing acute aortic dissection. Sci Rep 2017;7:12784.

13. Wang L, Zhang S, Xu Z, Zhang J, Li L, Zhao G. The diagnostic value of microRNA-4787-5p and microRNA-4306 in patients with acute aortic dissection. Am J Transl Res 2017;9:5138-49.

14. Li B, Wang Z, Hu Z, Zhang M, Ren Z, Zhou Z, et al. P38 MAPK signaling pathway mediates angiotensin II-induced miR143/145 gene cluster downregulation during aortic dissection formation. Ann Vasc Surg 2017;40:262-73.

15. Dorn LE, Petrosino JM, Wright P, Accornero F. CTGF/CCN2 is an autocrine regulator of cardiac fibrosis. J Mol Cell Cardiol 2018;121: 205-11.

16. Zhan B, Hu Z, Chen J, Zhu R, Zhao H, Yang J, et al. KLF15 overexpression protects $\beta$-aminopropionitrile-induced aortic rupture in rodent model via inhibiting connective tissue growth factor. Thorac Cardiovasc Surg 2017;65:120-5.

17. Frangogiannis NG. Matricellular proteins in cardiac adaptation and disease. Physiol Rev 2012;92:635-88.

18. Raffort J, Lareyre F, Clement M, Mallat Z. Micro-RNAs in abdominal aortic aneurysms: insights from animal models and relevance to human disease. Cardiovasc Res 2016;110:165-77.

19. Boon RA, Seeger T, Heydt S, Fischer A, Hergenreider E, Horrevoets AJ, et al. MicroRNA-29 in aortic dilation: implications for aneurysm formation. Circ Res 2011;109:1115-9.

20. Wu J, Song HF, Li SH, Guo J, Tsang K, Tumiati L, et al. Progressive aortic dilation is regulated by miR-17-associated miRNAs. J Am Coll Cardiol 2016;67:2965-77.

21. Elia L, Quintavalle M, Zhang J, Contu R, Cossu L, Latronico MV, et al. The knockout of miR-143 and -145 alters smooth muscle cell maintenance and vascular homeostasis in mice: correlates with human disease. Cell Death Differ 2009;16:1590-8.

22. Di Gregoli K, Mohamad Anuar NN, Bianco R, White SJ, Newby AC, George SJ, et al. MicroRNA-181b controls atherosclerosis and aneurysms through regulation of TIMP-3 and elastin. Circ Res 2017; 120:49-65.

23. Shen H, Lu S, Dong L, Xue Y, Yao C, Tong C, et al. hsa-miR-320d and hsa-miR-582, miRNA biomarkers of aortic dissection, regu- 
late apoptosis of vascular smooth muscle cells. J Cardiovasc Pharmacol 2018;71:275-82.

24. Chai KX, Chen YQ, Fan PL, Yang J, Yuan X. STROBE: the correlation of Cyr61, CTGF, and VEGF with polymyositis/dermatomyositis. Medicine (Baltimore) 2018;97:e11775.

25. Yan W, Liu H, Deng X, Jin Y, Wang N, Chu J. Acellular dermal matrix scaffolds coated with connective tissue growth factor accelerate diabetic wound healing by increasing fibronectin through PKC signalling pathway. J Tissue Eng Regen Med 2018;12:e1461-73.

26. Petrosino JM, Leask A, Accornero F. Genetic manipulation of CCN2/CTGF unveils cell-specific ECM-remodeling effects in injured skeletal muscle. FASEB J 2019;33:2047-57.

27. Kunzmann S, Ottensmeier B, Speer CP, Fehrholz M. Effect of pro- gesterone on Smad signaling and TGF- $\beta /$ Smad-regulated genes in lung epithelial cells. PLoS One 2018;13:e0200661.

28. Makino Y, Hikita H, Kodama T, Shigekawa M, Yamada R, Sakamori $\mathrm{R}$, et al. CTGF mediates tumor-stroma interactions between hepatoma cells and hepatic stellate cells to accelerate HCC progression. Cancer Res 2018;78:4902-14.

29. Zhou Y, Deng L, Zhao D, Chen L, Yao Z, Guo X, et al. MicroRNA-503 promotes angiotensin II-induced cardiac fibrosis by targeting Apelin-13. J Cell Mol Med 2016;20:495-505.

30. Wei C, Kim IK, Kumar S, Jayasinghe S, Hong N, Castoldi G, et al. NF$\kappa \mathrm{B}$ mediated miR-26a regulation in cardiac fibrosis. J Cell Physiol 2013;228:1433-42. 